\section{Spontaneous pneumothorax in a patient with an azygos lobe}

\author{
R T Sadikot, M E Cowen, A G Arnold
}

\begin{abstract}
The association between a spontaneous pneumothorax and an azygos lobe is surprisingly rare. A case is reported in which surgical management was difficult; it is suggested that thoracotomy is preferable to video-assisted thoracoscopic surgery in this situation. It is possible that the presence of an azygos lobe might protect against the subsequent development of a spontaneous pneumothorax, and the possible mechanism of this is discussed.

(Thorax 1997;52:579-580)
\end{abstract}

Keywords: spontaneous pneumothorax, azygos lobe.

Estimates of the incidence of an azygos lobe vary from $0.4 \%$ to $1 \%^{1}$ and its presence is usually assumed to be of no clinical significance. Similarly, spontaneous pneumothorax is relatively common with an incidence of more than seven cases in 100000 men and of more than one in 100000 women per annum. ${ }^{2}$ The association of a spontaneous pneumothorax with the presence of an azygos lobe ought therefore to occur reasonably often, though we can find only one previous report. ${ }^{3}$ We report a further case and discuss the problems that it posed for surgical management. Because of the apparent rarity of this association, we also discuss the possibility that the presence of an azygos lobe might somehow have a protective effect, thus reducing the incidence of spontaneous pneumothorax.

\section{Case report}

Medical Chest Unit R T Sadikot A G Arnold

Department of Thoracic Surgery M E Cowen

Castle Hill Hospital, Castle Road, Cottingham, North Humberside HU16 5JQ, UK

Correspondence to: Dr A G Arnold.

Received 17 November 1995 Returned to authors 14 February 1996

Revised version received 9 May 1996

Accepted for publication 22 May 1996 dyspnoea and a chest radiograph showed a large right pneumothorax and an azygos lobe (fig 1). The lung failed to expand adequately after the insertion of a chest drain, despite the use of suction. Because the lung had still not expanded after a period of two weeks of medical management the patient was referred to a thoracic surgical colleague. Video-assisted thoracoscopic surgery was attempted but dense pleural adhesions around the azygos fissure prevented access to the lung apex (area A, fig 2) which was necessary to inspect the lung for any air leak and to perform a parietal pleurectomy. Dissection of the adhesions was considered to be potentially hazardous because of the risk of bleeding from the azygos vein, thus ne-
A 20 year old male smoker presented with

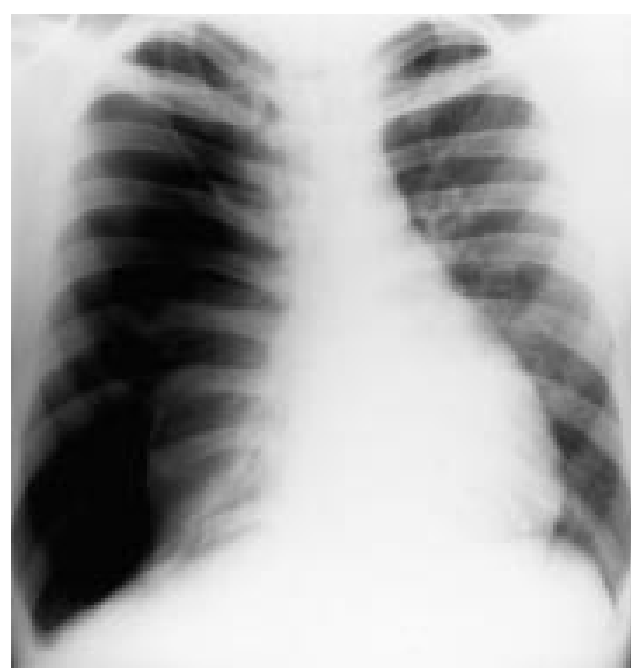

Figure 1 Chest radiograph showing a right pneumothorax and an azygos lobe.

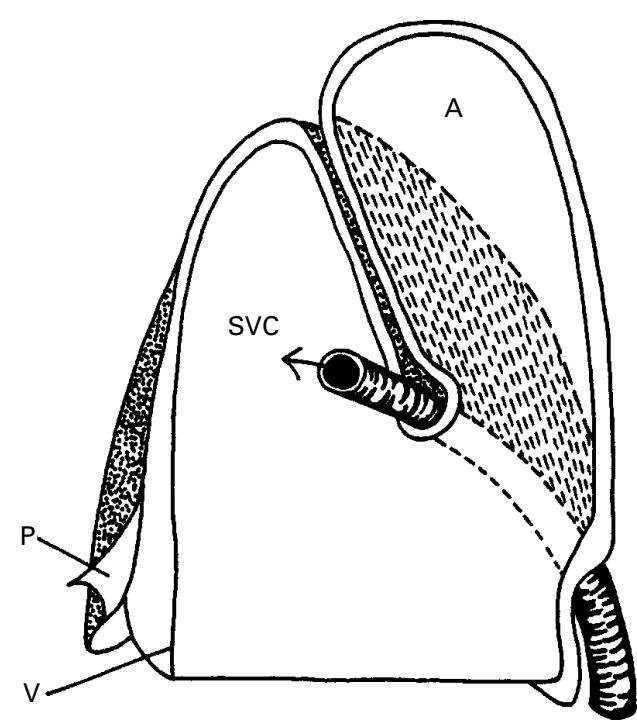

Figure 2 A diagrammatic representation of the abnormal anatomical structure of an azygos lobe. $A=$ lung apex; $P=$ parietal pleura; $V=$ visceral pleura; $S V C=$ superior vena cava.

cessitating a thoracotomy. At thoracotomy the azygos vein was divided and the parietal pleural reflections of the azygos fissure were resected, allowing access to the apex of the lung. No air leak was found and full parietal pleurectomy was performed. Apical bullae and blebs, such as those commonly encountered at the apices of the lungs in cases of primary spontaneous pneumothorax, were not seen. A postoperative radiograph showed full expansion of the lung. Subsequent review of the patient one year after surgery found that he had returned to normal health.

\section{Discussion}

The azygos lobe is a common malformation that was first described in $1777^{4}$ and has a reported incidence of $0.4-1 \% .^{1}$ The anatomical basis for this anomaly is a failure of normal 
migration of the azygos vein from the chest wall to its usual position in the tracheobronchial angle so that the invaginated visceral and parietal pleural layers persist to form a fissure. Since there is no corresponding alteration in the segmental architecture of the lung, the term "lobe" is a misnomer. It is usually supplied by branches of the apical segmental bronchus and is not unduly susceptible to disease. It is more commonly seen in men $^{5}$ and has been described on the left side. Familial occurrence of the anomaly has also been reported. ${ }^{6}$

Video-assisted thoracoscopic surgery is increasingly used for the management of a spontaneous pneumothorax, with recent reports suggesting its superiority to open thoracotomy. ${ }^{7}$ In our case the presence of an azygos lobe rendered this technique unsuccessful so that a conventional procedure was employed. Though a surgeon is rarely likely to encounter a patient with a spontaneous pneumothorax that has failed to resolve by medical management, we would suggest that the combination with an azygos lobe should be considered a relative contraindication to videoassisted thoracoscopic surgery and that an open thoracotomy should be considered.

Although the presence of an azygos lobe is usually of no clinical significance, effusions in the azygos fissure have been described. One previous publication ${ }^{3}$ described three cases of pneumothorax in association with an azygos lobe, one of which was spontaneous and the other two were iatrogenic. Two relatively common conditions might be expected to occur together by chance on a fairly regular basis. It is therefore surprising that no other reports of this association have been published. Discussion with numerous senior clinical and radiological colleagues seems to confirm the rarity of this association.

If the presence of an azygos lobe does somehow protect the patient from the subsequent development of a spontaneous pneumothorax, then the mechanism involved can only be the subject of speculation. Perhaps the double layer of reflected pleura becomes adherent, as in our case. This might follow the earlier development of a loculated effusion. Thus, any subsequent pneumothorax might be of limited size because of tethering, and perhaps therefore would be less likely to be diagnosed. Alternatively, it is possible that the altered anatomy at the apex of the lung in a patient with an azygos lobe somehow protects the apex from the development of the blebs and bullae that are characteristically seen in those young adults who present with a spontaneous pneumothorax. ${ }^{8}$ These were not seen in our case.

Another explanation might relate to the distribution of mechanical stresses within the lung which are not uniform but are much higher towards the apex of the lung..$^{9}$ The uneven distribution of stress results from distortion of the lung by its own weight, and experimental models have shown that the vectors are as great in the lateral as in the vertical planes. Transmission of transient forces to the apex of the lung has been proposed as a factor in the development of a spontaneous pneumothorax. ${ }^{9}$ The four layers of pleura forming the azygos lobe, acting as a septum, may protect the apex of the lung from the transmission of such forces in both the lateral and vertical planes because of its oblique position relative to the lung apex.

The rarity of the association between spontaneous pneumothorax and an azygos lobe may provide a clue as to the mechanisms involved in the aetiology of the development of spontaneous pneumothorax.

Figure 2 is a modification of an illustration take from Respiratory medicine (2nd edition) and is reproduced by the kind permission of Dr R A L Brewis and WB Saunders Company Ltd. We would also like to thank Catherine Turner for secretarial support.

1 Boyden EA. The distribution of bronchi in gross abnormalities of the right upper lobe, particularly lobes subdivided by the azygos vein and those containing predivided by the azygos vein and those cont
eparterial bronchi. Radiology 1952;58:797-807.

2 Melton LJ, Hepper NGG, Offord KP. Incidence of spontaneous pneumothorax in Olmsted County, Minnesota 1950-1974. Am Rev Respir Dis 1979;120:1379-82.

3 Weissman JL, Austin JHM. Pneumothorax and an azygos lobe. $\mathcal{F}$ Thorac Imaging 1989;4:VI-IX

4 Wrisberg HA. Observationes anatomicae de vena azyga duplici, aliisque hujus venae varietatibus. Novis Commentariis Societatis Reg. Scient., Göttingen Anno 1777, Tome VIII 14.

5 Fisher MS. Adam's lobe. Radiology 1985;154:547.

6 Postmus PE, Kerstjens JM, Breed A, Jaut EVD. A family with lobus venae azygos. Chest 1986;90:298-9.

7 Waller DA, Forty J, Moritt GM. Video-assisted thoracoscopic surgery versus thoracotomy for spontaneous pneumothorax. Ann Thorac Surg 1994;58:372.

8 Ohata M, Suzuki H. Pathogenesis of spontaneous pneumothorax - with special reference to the ultrastructure of thorax - with special reference to the ultrast
emphysematous bullae. Chest 1980;77:771-6.

9 West JB Distribution of mechanical stress in the lung, possible factor in localisation of pulmonary disease. Lancet $1971 ; \mathrm{i}: 839-41$. 